\title{
Mesure par conductimétrie de la réceptivité aux maladies fongiques et du niveau de résistance induite par élicitation. Étude sur 3 modèles expérimentaux
}

\author{
PM Molot 1, P Mas 1, V Pivot 2, M Bruneteau 2, T Staron 3, H Ferrière 1, JM Lemaire 1 \\ 1 INRA, station de pathologie végétale, BP 94, 84143 Montfavet Cedex; \\ 2 Université Claude Bernard, laboratoire de biochimie microbienne, 43, bd du 11 novembre, 69622 Villeurbanne Cedex; \\ 3 INRA, station des antibiotiques et des bioconversions, route de Thiverval, 78850 Thiverval-Grignon, France
}

(Reçu le 10 juin 1991; accepté le 16 octobre 1991)

\begin{abstract}
Résumé - L'appréciation des dégâts parasitaires, souvent difficile à évaluer, peut être effectuée par des mesures conductimétriques; en effet, les cellules altérées libèrent davantage d'électrolytes que les cellules saines dans le milieu extérieur, dont on mesure la conductance. Cette méthode peut être utilisée dans différents cas, notamment lorsqu'il y a attaque au niveau racinaire. On mesure alors la conductance de l'eau dans laquelle baignent des racines contaminées (piment - Phytophthora capsici, tableau I), ou des feuilles appartenant à des plantes dont le système racinaire est malade (blé - Gaeumannomyces graminis) (tableau II).

Les mesures de perte d'électrolytes par conductimétrie apportent également des renseignements intéressants sur la réceptivité aux maladies des tissus sains; celle-ci varie en particulier avec l'âge des tissus, notamment en fonction de la hauteur des entre-nœuds et des étages foliaires; elle peut être mesurée par la quantité d'ions relargués, car une bonne corrélation existe entre les notes de sensibilité à la maladie et la conductance des tissus sains. Les études ont été conduites, d'une part, sur le couple maïs - Helminthosporium turcicum (tableau III avec $r=+0,953$ ) et d'autre part, sur le couple piment - Phytophthora capsici (tableau IV avec $r=+0,909$ pour les feuilles et $r=+0,932$ pour les tiges).

Les éliciteurs ont la propriété d'induire chez les végétaux des mécanismes de défense. Le passage à l'état élicité se traduit par une modification de la perméabilité cellulaire qui a pu être observée ici avec 2 éliciteurs phospholipidiques : un sphingophospholipide à inositol issu de $P$ capsici et une phosphatidylcholine issue de $G$ graminis. L'étude sur piment, qu'il s'agisse d'élicitation directe ou d'élicitation à distance (tableau V), montre que les tissus traités relarguent de moins en moins d'électrolytes au fur et à mesure que la concentration en éliciteur augmente et que la protection se renforce. II existe cependant une concentration en éliciteur optimale au-delà de laquelle l'effet s'inverse. Chez le blé, les semences ayant germé en présence d'éliciteur donnent des plantules qui libèrent moins d'électrolytes que les témoins (tableau VI). L'intérêt de cette méthode en phytopathologie est discuté.
\end{abstract}

Phytophthora capsici / Gaeumannomyces graminis / Helminthosporium turcicum / relargage d'ions / éliciteur / sphingophospholipide à inositol / phosphatidylcholine / réceptivité tissu sain / capsicum = piment / Triticum = blé $/$ Zea mays = maïs $/$ perméabilité cellulaire

Summary - Measurement of electrolyte leakage to assess host susceptibility and elicitor-induced resistance: study of 3 experimental models. Damage to plants due to parasites is often difficult to evaluate, particularly in root system diseases. When altered cells (necrotic or with altered metabolism) are soaked in water, they leak electrolytes and the conductance of the surrounding solution increases. For example, the amount of electrolytes released by pepper roots attacked by Phytophthora capsici increases with zoospore concentration (table 1); likewise, at flowering time, leaves of wheat (when floated on water) contaminated by Gaeumannomyces graminis have a higher conductance than the healthy control (table II). The susceptibility of healthy tissues changes with age, particularly with the height of internodes and foliar position above the soil. In the corn - Helminthosporium turcicum (table III) and pepper - $\mathrm{P}$ capsici system interactions (table IV), there is a good correlation between the conductance of healthy tissues and the susceptibility ratings of corresponding tissues (corn leaf, $\mathrm{r}=0.953$; pepper leaf, $\mathrm{r}=0.909$; pepper stem, $\mathrm{r}=0.932$ ). Elicitation may be performed either by soaking detached cotyledons or by truncating and soaking non-detached cotyledons (pepper, table $V$ ) or by soaking the seeds (wheat, table VI). Two phospholipid elicitors are used: inositol sphingophospholipid isolated from P capsici and phosphatidylcholine isolated from G graminis. The elicitor amount of elec- 
trolytes leaked by plants decreases whith increasing concentrations. However, there is an optimum concentration (between 5 and $10 \mu \mathrm{g} / \mathrm{ml}$ ) beyond which the effect is reversed.

Phytophthora capsici / Gaeumannomyces graminis / Helminthosporium turcicum / electrolyte leakage / elicitors / inositol sphingophospholipid and phosphatidylcholine / healthy tissue receptivity / Capsicum = pepper / Triticum $=$ wheat $/$ Zea mays $=$ corn $/$ cellular permeability

\section{INTRODUCTION}

Confrontées à une attaque parasitaire, les plantes présentent non seulement des différences de sensibilité variétale mais aussi, pour un même génotype, un comportement susceptible de varier selon l'âge et les conditions du milieu.

L'appréciation des dégâts causés par une maladie s'effectue généralement par des notations où le "coup d'œill» de l'observateur, souvent très subjectif, joue un rôle déterminant. Or il est connu que l'altération des tissus par les champignons, que ce soit par invasion du mycélium ou par action des toxines, entraîne une libération d'électrolytes (Halloin et al, 1973; Cahill et al, 1985; Pavlovkin et al, 1986; Zilberstein et Pinkas, 1987; Molot et al, 1987; Rosati et al, 1988). On conçoit donc qu'un changement de la résistance du milieu extérieur, c'est-à-dire de l'eau dans laquelle trempe l'organe malade, puisse donner une indication précise de l'importance des dégâts.

Au cours de la présente étude, notre objectif sera tout d'abord de mettre en parallèle les notes d'appréciation visuelle du matériel contaminé et les valeurs de conductance (inverse de la résistance) de ce même matériel. Nous prendrons successivement des racines de piment contaminées par Phytophthora capsici et des feuilles de blé non directement parasitées, mais appartenant à une plante dont les racines sont colonisées par Gaeumannomyces graminis.

Par ailleurs, il est bien connu en phytopathologie que le contact d'une plante hôte et de son parasite ne constitue pas une condition suffisante pour la réalisation de l'infection. À ce moment précis, le végétal doit se trouver dans une situation particulière, appelée état de réceptivité, qui peut varier en fonction de nombreux facteurs: âge de la plante, état de la végétation, mode de croissance, nature des surfaces externes, composition des tissus, etc. Cette notion demeure difficile à appréhender.

À la suite de travaux sur la réponse hypersensible du tabac (Atkinson et al, 1990), nous avons pensé qu'entre réceptivité et perméabilité cellu- laire d'un tissu sain, pouvait exister une relation. Cette hypothèse a été confrontée à 2 modèles expérimentaux : maïs-Hehminthosporium turcicum (feuilles) et piment- $P$ capsici (feuilles et tiges), sur lesquels nous avons tenté, par des mesures conductimétriques, d'apprécier la réceptivité des tissus sains aux maladies.

Enfin, la réceptivité pouvant être modulée par action d'un éliciteur, il nous a paru intéressant de vérifier si, dans le cas d'une protection accrue du végétal (résistance induite), il y a rétention ou, au contraire, libération d'ions dans le milieu extracellulaire. Notre étude s'appuiera ici sur deux couples : piment- $P$ capsici et blé-G graminis.

\section{MATÉRIEL ET MÉTHODES}

\section{Matériel végétal et cryptogamique}

Nous avons expérimenté sur piment (variété «Bastidon») contaminé par Phytophthora capsici et sur les couples maïs (variété "Carola") - Helminthosporium turcicum et blé (variété "Lutin") - Gaeumannomyces graminis, cette dernière variété ayant été choisie parce que particulièrement apte à la prémunition (Lucas et al, 1986). Tous les essais sont effectués sur du matériel semé et cultivé en serre.

\section{Élicitation du matériel végétal}

Deux éliciteurs phospholipidiques purs ont été utilisés: un sphingophospholipide à inositol (Lhomme, 1988; Lhomme et al, 1990) issu du mycélium de Phytophthora capsici et une phosphatidylcholine isolée par Th Staron et issue du mycélium de la souche hypoagressive 911 de $G$ graminis (Molot et al, 1989). Ces 2 substances sont employées en solution dans l'eau à des concentrations comprises entre 1 et $100 \mu \mathrm{g} / \mathrm{ml}$. Elles sont mises directement au contact de l'organe étudié : trempage de $20 \mathrm{~min}$ dans l'éliciteur des cotylédons ou des hypocotyles de piment détachés et mise en attente $24 \mathrm{~h}$ en chambre humide. L'élicitation peut également être réalisée soit par imbibition de $48 \mathrm{~h}$ de la semence de céréales dans l'inducteur, soit par trempage extemporané dans l'éliciteur d'une extrémité tronquée de cotylédon de piment non détaché de la plante (Molot et al, 1989). Dans tous les 
cas, un délai minimal d'attente de $24 \mathrm{~h}$ (entre élicitation et poursuite de l'expérimentation) est respecté.

\section{Contaminations artificielles}

\section{Avec le couple piment - P capsici}

Le champignon cultivé sur milieu V8 est utilisé soit sous forme de mycélium, soit sous forme de zoospores produites dans des conditions décrites par ailleurs (Molot et al, 1976). Le mycélium sert à contaminer les plantes adultes, qu'il s'agisse de tiges décapitées (Pochard et al, 1976) ou de feuilles en survie sur l'eau et blessées sur lesquelles on dépose un implant de culture du champignon (Molot et al, 1980a). On utilise les zoospores pour la contamination des racines ou des cotylédons de plantules au stade 2 cotylédons bien étalés.

\section{Racines}

Les plantules sont arrachées; leur système racinaire est lavé, trempé $1 \mathrm{~h}$ dans une suspension de zoospores nageuses titrant de 100 à 5000 zoospores $/ \mathrm{ml}$, puis remis à incuber dans de l'eau à $20^{\circ} \mathrm{C}$ pendant $4 \mathrm{j}$. Les racines immergées sont maintenues à l'obscurité, tandis que la partie aérienne reçoit l'éclairage naturel.

\section{Cotylédons}

Détachés de la plantule et mis en survie sur l'eau en boîte de Petri ( 20 cotylédons par boîte), ils sont contaminés par dépôt, à la face inférieure du limbe, de $10 \mu \mathrm{l}$ d'une suspension à 75000 zoospores enkystées par $\mathrm{ml}$. L'incubation se fait à $20^{\circ} \mathrm{C}$ sous éclairage naturel pendant $5 \mathrm{j}$.

Sur plantes adultes contaminées, les notations consistent à mesurer les longueurs de tiges nécrosées (plantes en place) ou les diamètres de zone foliaire brune (feuilles en survie sur l'eau). Sur plantules, on attribue aux racines et aux cotylédons des notes visuelles de 0 (pas d'attaque) à 5 (attaque maximale); sur cotylédons détachés, les notes cumulées sont traduites en indices de maladie (Molot et al, 1980b).

\section{Dans le cas de l'interaction blé - G graminis}

La contamination est effectuée à l'aide de grains d'orge infectés et secs que l'on dispose à proximité de la semence mise en terre (Lucas et al, 1986). Ces essais sont réalisés en pots.

\section{Avec le couple maïs $-\boldsymbol{H}$ turcicum}

L'inoculum est préparé à partir de cultures fongiques développées à l'obscurité pendant deux semaines : après avoir ajouté un peu d'eau stérile, on racle la surface de la culture et on ajuste la concentration à 20000 spores par $\mathrm{ml}$. Cette suspension est pulvérisée sur la plante entière, juste avant la sortie de la pani- cule. Les maïs sont alors placés en atmosphère humide, sous serre. Les notations de symptômes se font de 0 à 5 selon l'importance des taches foliaires.

\section{Mesures de la perméabilité cellulaire des tissus par conductimétrie}

Le matériel végétal destiné aux mesures est issu de jeunes plantules ou de plantes adultes.

Sur jeunes plantules, dans le cas du piment, il est constitué de racines, de cotylédons, de tronçons de d'hypocotyles, dans le cas du blé, des 3 premières feuilles.

Sur plantes adultes, les échantillons peuvent être des feuilles entières (piment), des tronçons de feuilles (blé, maïs), des fragments d'entre-nœuds de tige (piment). Les prélèvements sont effectués à tous les niveaux de la plante.

Chaque lot est pesé (en moyenne $250 \mathrm{mg}$ ) et immergé dans $100 \mathrm{ml}$ d'eau bidistillée où il demeure à $22{ }^{\circ} \mathrm{C}$ sans agitation. L'augmentation de conductance de l'eau dans laquelle baigne le matériel végétal est appréciée à l'aide d'un conductimètre Metrohm Herisau $E 382$ (constante de l'électrode 0,89). La conductance (inverse de la résistance) donnée en $\mu \mathrm{S}$ traduit l'importance du relargage des ions tissulaires dans le milieu extérieur. Elle fait l'objet de plusieurs lectures dans le temps dont seule la dernière, généralement effectuée au bout de $48 \mathrm{~h}$, figure dans nos résultats.

Chaque traitement comporte 5 répétitions correspondant à 5 plantes différentes. Des précisions expérimentales, spécifiques à certains essais, seront fournies avec les résultats.

\section{RÉSULTATS}

\section{Appréciation de l'intensité des dégâts parasitaires}

Cette approche a pu être réalisée à l'aide de 2 modèles : l'un concernant le couple piment $P$ capsici où il s'agit d'évaluer l'importance de fines nécroses racinaires réparties de manière très hétérogène, l'autre le couple blé - G graminis où la présence du champignon localisé dans la partie inférieure de la plante entraîne des perturbations du système aérien, difficiles à analyser et à noter.

\section{Attaque parasitaire au niveau des racines. Modèle piment - $P$ capsici (tableau I)}

Les plantules de piment sont arrachées et remises à tremper dans l'eau après contamination de leurs racines. 
Tableau I. Contamination des racines de piment "Bastidon" par une suspension de zoospores de $P$ capsici à différentes concentrations. Appréciation des attaques racinaires par 2 voies d'approche. Les racines sont prélevées sur plantules au stade 2 cotylédons, trempées dans l'inoculum et mises à incuber à $20^{\circ} \mathrm{C}$ soit dans l'eau (note de sensibilité), soit en chambre humide (conductance).

\begin{tabular}{ccc}
\hline $\begin{array}{c}\text { Concentration } \\
\text { en zoospores } / m l\end{array}$ & $\begin{array}{c}\text { Note } \\
\text { de sensibilité }\end{array}$ & $\begin{array}{c}\text { Conductance } \\
(\mu S)\end{array}$ \\
\hline 0 & 0 & 6,62 \\
100 & 0,3 & 10,54 \\
500 & 1 & 26,02 \\
1000 & 2 & 44,24 \\
5000 & 5 & 149,20 \\
\hline
\end{tabular}

Les symptômes apparaissent progressivement sous forme de petites nécroses irrégulières difficiles à apprécier; nous leur avons néanmoins attribué une note dont la valeur augmente avec la concentration en zoospores de l'inoculum.

En ce qui concerne les mesures de conductance, les racines sont prélevées le jour même de la notation visuelle, pesées et replacées dans $100 \mathrm{ml}$ d'eau dont on mesure, dans le temps, la résistance. Entre les notes de sensibilité et les valeurs de conductance, il existe une très bonne corrélation : $r=+0,991$.

\section{Répercussions sur le feuillage d'une attaque racinaire. Modèle blé - G graminis (tableau II)}

Un semis de blé Lutin est effectué en pots au contact de grains contaminés par $G$ graminis. En fin de montaison, on note des différences difficilement chiffrables entre lots sains et lots malades. L'avant-dernière feuille placée sur eau révèle rapidement, après mesure de conductance, des différences importantes entre plantes parasi-

Tableau II. Conductance en $\mu \mathrm{S}$ de l'avant dernière feuille de blé «Lutin» après contamination des racines par $G$ graminis. 5 plantes en fin de montaison, contamination par $G$ graminis au semis.

\begin{tabular}{lrrrrrr}
\hline & \multicolumn{1}{c}{ II } & III & IV & $V$ & Moy \\
\hline Témoin sain & 4,9 & 9,0 & 6,1 & 7,5 & 7,7 & 7,04 \\
Contaminé & 18,2 & 23,1 & 25,7 & 20,6 & 21,4 & 21,80
\end{tabular}

tées et témoins. Bien que l'attaque du champignon au niveau des racines soit difficile à déceler, on constate que les feuilles des plantes atteintes ont une conductance environ 3 fois plus forte que celle des feuilles des plantes témoins.

\section{Appréciation, sur du matériel sain, du niveau de réceptivité à une attaque parasitaire}

Des observations préliminaires montrant que l'intensité des attaques parasitaires varie en fonction de la hauteur des organes au-dessus du sol, nous avons choisi 2 modèles pour préciser ce phénomène.

\section{Modèle maïs - Helminthosporium turcicum (tableau III)}

Cultivé en serre et contaminé par pulvérisation de spores juste avant la sortie de la panicule, le maïs présente sur feuilles des taches elliptiques, parallèles aux nervures, généralement installées dans la partie médiane du limbe. Elles sont faciles à dénombrer. Il est donc possible d'apprécier l'intensité de l'attaque pour chaque étage foliaire. On constate que les feuilles de la base sont plus sévèrement atteintes que les feuilles du sommet.

Sur des plantes témoins, cultivées dans des conditions identiques, on procède, étage par étage, à des mesures conductimétriques des fragments de limbe immergés dans l'eau. Les feuilles de la base relarguent plus d'ions que celles du sommet, la diminution se faisant progressivement du bas vers le haut. Pour chaque

Tableau III. Sensibilité des feuilles de maïs "Carola" à $H$ turcicum en fonction de l'étage foliaire et relation avec la conductance des tissus sains. Les prélèvements sont effectués juste avant la sortie de la panicule.

\begin{tabular}{lcc}
\hline $\begin{array}{c}\text { Étage foliaire } \\
\text { (numéroté } \\
\text { de bas en haut) }\end{array}$ & $\begin{array}{c}\text { Sensibilité } \\
\text { aH turcicum } \\
\text { (de } 0 \text { à 5) }\end{array}$ & $\begin{array}{c}\text { Conductance } \\
\text { des tissus sains } \\
(\mu S)\end{array}$ \\
\hline & & \\
7 (haut) & 1,3 & 6,63 \\
6 & 1,9 & 6,89 \\
5 & 2,2 & 7,14 \\
4 & 2,8 & 7,81 \\
3 & 3,7 & 7,91 \\
2 & 4,1 & 9,50 \\
1 (bas) & 4,8 & 10,01 \\
\hline
\end{tabular}


étage foliaire, entre les notes moyennes de sensibilité et les valeurs moyennes de conductance obtenues sur 5 plantes différentes, le coefficient de corrélation est de $+0,953$.

\section{Modèle piment - Phytophthora capsici (tableau IV)}

Les contaminations artificielles sont réalisées sur plantes décapitées à différents niveaux (Pochard et al, 1976), tandis que les feuilles des différents étages sont prélevées, mises en survie en boîte de Petri et inoculées par le parasite (Molot et al, 1980a).

Pour les feuilles, on constate, comme pour le maïs (tableau III), une diminution progressive de la sensibilité à $P$ capsici des feuilles de la base (très sensibles) vers celles du sommet (peu sensibles).

Sur tiges, l'hypocotyle se révèle très sensible mais la sensibilité des tissus va en diminuant jusqu'au troisième entre-nœud; au-delà de ce niveau et au fur et à mesure que les contaminations sont effectuées de plus en plus haut, on constate une sensibilité croissante des tissus.

Ces notations de symptômes peuvent être mises en parallèle avec les mesures de conductance pratiquées sur tissu sain de la même façon que pour le modèle précédent; une forte sensibilité se traduit par des conductances élevées, une moindre sensibilité par des conductances faibles. Les coefficients de corrélation sont respectivement de $+0,909$ pour les feuilles et de $+0,932$ pour les tiges.

\section{Variations de la conductance des tissus sains sous l'effet de l'élicitation}

\section{Modèle piment (tableau V)}

Sur jeunes plantules (stade 2 cotylédons), nous pratiquons l'élicitation sur organes détachés ou sur organes en place.

\section{Élicitation sur organes détachés}

Les cotylédons ou les hypocotyles (tronçons de $2 \mathrm{~cm}$ situés juste sous les cotylédons) sont prélevés, trempés 20 min dans l'éliciteur, puis placés $24 \mathrm{~h}$ en chambre humide avant la mesure de conductance.

Quelques cotylédons subissent en outre une contamination artificielle pour contrôler le niveau de résistance induite. Les valeurs de conductance, toujours effectuées sur tissu sain immergé dans l'eau, varient avec la concentration en éliciteur. Relativement élevées aux fortes et faibles concentrations, elles présentent un minimum pour des quantités d'éliciteur comprises entre 2,5 et $10 \mu \mathrm{g} / \mathrm{ml}$.

Tableau IV. Sensibilité des feuilles et des tiges de piment «Bastidon» à $P$ capsici en fonction du niveau au-dessus du sol et relation avec la conductance des tissus sains. Les prélèvement sont effectués juste avant la floraison. - : mesure non effectuée. Chaque traitement comporte 5 répétitions correspondant à 5 plantes différentes.

Niveau étudié

( $n^{\circ}$ entre-nœud)
Sensibilité à $\mathbf{P}$ capsici

Feuilles

Tiges

(longueur nécrose en $\mathrm{mm}$ )

(diamètre nécrose en $\mathrm{mm}$ )

Conductance des tissus sains $(\mu S)$

Tiges Feuilles

$\begin{array}{cccrr}9 \text { (haut) } & 43,0 & 29,2 & 158,72 & 7,61 \\ 8 & 42,2 & 33,4 & 141,02 & 9,22 \\ 7 & 40,4 & 34,6 & 131,58 & 12,10 \\ 6 & 37,8 & 38,8 & 112,24 & 12,60 \\ 5 & 36,6 & 40,8 & 84,76 & 16,77 \\ 4 & 35,4 & 41,6 & 63,76 & 18,91 \\ 3 & 35,0 & 42,6 & 61,28 & 25,50 \\ 2 & 38,0 & 44,0 & 70,40 & -26,80 \\ 1 & 38,4 & - & 84,06 & - \\ \text { niveau } & & & & \\ \text { cotylédonnaire } & & - & 90,9 & \\ 0 & 40,3 & & & \end{array}$


Tableau V. Effet de l'élicitation sur la conductance des tissus sains et la sensibilité des cotylédons du piment "Bastidon» à $P$ capsici. L'élicitation est pratiquée soit par trempage de l'organe détaché dans l'éliciteur (1), soit par excision de l'extrémité des cotylédons en place suivie d'un trempage extemporané dans l'éliciteur (2). SPI = phospholipide à inositol (sphigophospholipide). $\mathrm{PC}=$ phospholipide à choline (phosphatidylcholine). Les conductances sont exprimées en $\mu \mathrm{S}$ et les indices de maladie en \%.

\begin{tabular}{|c|c|c|c|c|c|c|c|}
\hline \multirow{3}{*}{$\begin{array}{l}\text { Concentration } \\
\text { en éliciteur } \\
(\mu \mathrm{g} / \mathrm{ml})\end{array}$} & \multicolumn{4}{|c|}{ Organes détachés (1) } & \multicolumn{3}{|c|}{ Plantes en place (2) } \\
\hline & \multirow{2}{*}{$\begin{array}{c}\text { Nature } \\
\text { de } \\
\text { l'éliciteur }\end{array}$} & \multirow{2}{*}{$\begin{array}{l}\text { Hypocotyles } \\
\text { Conductance }\end{array}$} & \multicolumn{2}{|c|}{ Cotylédons } & \multirow{2}{*}{$\begin{array}{l}\text { Hypocotyles } \\
\text { Conductance }\end{array}$} & \multicolumn{2}{|c|}{ Cotylédons } \\
\hline & & & Conductance & $\begin{array}{l}\text { Indice } \\
\text { de maladie }\end{array}$ & & Conductance & Indice de maladie \\
\hline 0 & & 3,12 & 9,50 & 80,3 & 4,60 & 13,51 & 99,8 \\
\hline 1 & $\begin{array}{l}\text { SPI } \\
\text { PC }\end{array}$ & $\begin{array}{l}2,01 \\
3,07\end{array}$ & $\begin{array}{l}7,29 \\
8,49\end{array}$ & $\begin{array}{l}71,5 \\
75,1\end{array}$ & $\begin{array}{l}3,36 \\
4,56\end{array}$ & $\begin{array}{l}11,76 \\
13,16\end{array}$ & $\begin{array}{l}95,4 \\
99,0\end{array}$ \\
\hline 2,5 & $\begin{array}{l}\text { SPI } \\
\text { PC }\end{array}$ & $\begin{array}{l}2,40 \\
2,89\end{array}$ & $\begin{array}{l}6,92 \\
7,05\end{array}$ & $\begin{array}{l}68,0 \\
72,2\end{array}$ & $\begin{array}{l}3,25 \\
4,54\end{array}$ & $\begin{array}{l}10,85 \\
13,04\end{array}$ & $\begin{array}{l}59,9 \\
91,2\end{array}$ \\
\hline 5 & $\begin{array}{l}\text { SPI } \\
\text { PC }\end{array}$ & $\begin{array}{l}2,71 \\
2,90\end{array}$ & $\begin{array}{l}6,35 \\
6,01\end{array}$ & $\begin{array}{l}53,9 \\
49,4\end{array}$ & $\begin{array}{l}3,30 \\
4,32\end{array}$ & $\begin{array}{l}12,09 \\
12,29\end{array}$ & $\begin{array}{l}70,1 \\
81,9\end{array}$ \\
\hline 10 & $\begin{array}{l}\text { SPI } \\
\text { PC }\end{array}$ & $\begin{array}{l}3,02 \\
2,96\end{array}$ & $\begin{array}{l}5,75 \\
6,02\end{array}$ & $\begin{array}{l}35,7 \\
41,2\end{array}$ & $\begin{array}{l}3,64 \\
4,16\end{array}$ & $\begin{array}{l}12,58 \\
11,71\end{array}$ & $\begin{array}{l}75,1 \\
68,2\end{array}$ \\
\hline 50 & $\begin{array}{l}\text { SPI } \\
\text { PC }\end{array}$ & $\begin{array}{l}3,03 \\
3,02\end{array}$ & $\begin{array}{l}6,81 \\
7,17\end{array}$ & $\begin{array}{l}45,5 \\
51,0\end{array}$ & $\begin{array}{l}4,14 \\
4,62\end{array}$ & $\begin{array}{l}13,21 \\
14,43\end{array}$ & $\begin{array}{l}77,9 \\
75,7\end{array}$ \\
\hline 100 & $\begin{array}{l}\text { SPI } \\
\text { PC }\end{array}$ & $\begin{array}{l}3,27 \\
3,08\end{array}$ & $\begin{array}{l}7,29 \\
7,41\end{array}$ & $\begin{array}{l}54,9 \\
60,4\end{array}$ & $\begin{array}{l}4,87 \\
5,05\end{array}$ & $\begin{array}{l}14,19 \\
16,32\end{array}$ & $\begin{array}{l}87,0 \\
93,2\end{array}$ \\
\hline
\end{tabular}

L'indice de maladie des cotylédons évolue de la même manière. Les coefficients de corrélation entre les conductances des cotylédons sains et les indices de maladie des cotylédons infectés ont été calculés pour chaque éliciteur:

- sphingophospholipide à inositiol $: r=+0,820$

- phosphatidylcholine : $r=+0,868$

La corrélation apparaît particulièrement bonne dans le cas de cette dernière substance.

II ressort qu'aux faibles et fortes concentrations en éliciteur les plantules de piment sont plus sensibles à la maladie et ont une conductance plus élevée qu'aux concentrations moyennes.

\section{Élicitation sur organes en place}

Sur chaque plantule, l'élicitation est pratiquée à l'extrémité tronquée d'un seul cotylédon. Au bout de $24 \mathrm{~h}$, on prélève le cotylédon opposé et l'hypocotyle pour mesurer le relargage d'ions des tissus sains sur eau.
Comme précédemment, quelques cotylédons détachés non directement élicités sont contaminés artificiellement pour apprécier le niveau de protection.

Les conductances atteignent des valeurs plus élevées que sur organes détachés, traduisant une tendance à libérer davantage d'ions; elles présentent un minimum pour des concentrations en éliciteur voisines de $5 \mu \mathrm{g} / \mathrm{ml}$.

Par ailleurs, les cotylédons apparaissent plus sensibles que dans le cas précédent avec, cependant, un effet de protection se manifestant pour des concentrations moyennes en éliciteur.

Entre les conductances des cotylédons sains et les indices de maladie des cotylédons infectés, les coefficients de corrélation sont respectivement de $+0,572$ pour le sphingophospholipide à inositol et de $+0,373$ pour la phosphatidylcholine.

II apparaît sur organes en place une moindre efficacité pour l'induction de résistance de l'élicitation à distance par rapport à l'élicitation in situ 
(expérience précédente). De plus, des 2 éliciteurs utilisés, le sphingophospholipide à inositol issu du parasite présente vis-à-vis du piment la meilleure spécificité : effet de protection plus accentué, conductance plus faible.

\section{Modèle blé (tableau VI)}

Après imbibition de la semence pendant $48 \mathrm{~h}$ à $20^{\circ} \mathrm{C}$ dans l'éliciteur, le semis est effectué en pot sur sol sain. Les prélèvements de jeunes feuilles entières sont effectués 14 j après le semis, au stade d'apparition de la troisième feuille. Les mesures de conductance révèlent des valeurs minimales pour des concentrations en éliciteur comprises entre 5 et $10 \mu \mathrm{g} / \mathrm{ml}$; aux fortes doses, le relargage des ions est plus important et peut même dépasser le témoin non élicité dans le cas du sphingophospholipide à inositol.

On remarquera que, contrairement à ce qui se passe pour le piment, la phosphatidylcholine issue de $G$ graminis entraîne chez le blé une plus forte rétention des ions que le sphingophospholipide à inositol issu de $P$ captici.

\section{DISCUSSION ET CONCLUSION}

Au terme de cette étude, il apparaît que les mesures de perméabilité cellulaire permettent tout d'abord d'évaluer de façon précise le niveau d'altération des plantes parasitées par un champignon. Lorsqu'il s'agit d'organes comme les racines, où le "chevelu» est souvent attaqué de manière irrégulière, l'appréciation visuelle n'est

Tableau VI. Conductance $(\mu \mathrm{S})$ des trois premières feuilles de blé "Lutin" après élicitation par imbibition de la semence. SPI = phospholipide à inositol (sphingophospholipide) $. \mathrm{PC}=$ phospholipide à choline (phosphatidylcholine). Mesures effectuées 14 j après le semis. Chaque traitement comporte 5 répétitions correspondant à 5 plantes différentes.

Concentration en éliciteur
$(\mu g / m l)$$\quad S P I \quad P C$

$\begin{array}{rll}0 & 3,01 & 3,08 \\ 1 & 2,94 & 2,93 \\ 5 & 2,83 & 2,74 \\ 10 & 2,88 & 2,67 \\ 50 & 3,01 & 2,76 \\ 100 & 3,15 & 2,97\end{array}$

pas toujours aisée. Différents auteurs ont abordé ce problème par des mesures conductimétriques, notamment sur le modèle carotte - Sclerotinia sclerotiorum (Newton et al, 1973), pois Fusarium solani f $\mathrm{sp}$ pisi ou phaseoli (Kendra et Hadwiger, 1987), avocatier - $P$ cinnamomi (Zilberstein et Pinkas, 1987). Dans chaque cas, on observe une augmentation de la conductance avec le degré d'altération tissulaire.

A notre connaissance, nous sommes les premiers à avoir montré, chez le blé, que les mesures de conductance révèlent des différences significatives entre plante saine et plante malade, même si le parasite n'est pas présent dans l'organe testé de la plante contaminée.

Les observations effectuées au champ en zone naturellement infectée par Helminthosporium turcicum (Molot, 1968) sont en accord avec les résultats obtenus en conditions expérimentales. La maladie ne s'observe jamais sur jeunes plantules; les premières nécroses n'apparaissent qu'à partir du stade 7-8 feuilles et continuent à se développer jusqu'à la maturité; leur évolution s'effectue de la base (très attaquée) vers le sommet de la plante (peu attaqué), cette constatation étant généralement attribuée à des causes épidémiologiques. Sans exclure cette hypothèse, nous avons démontré qu'il existe chez le maiis une prédisposition constitutive en fonction de l'âge des tissus, mesurable de façon simple par conductimétrie.

De la même manière, en expérimentant sur piment juste avant la floraison, par prélèvement "systématique" de la base au sommet (donc sur des tissus d'âge décroissant), nous avons pu mettre en évidence une relation entre la sensibilité des tissus à $P$ capsici et leur capacité à relarguer des électrolytes. Cette sensibilité apparaît élevée sur tiges jeunes (sommet de plante) et sur tiges vieilles (base de plante), alors qu'elle atteint sa plus faible valeur en zone moyenne. Établie par des contaminations à plusieurs niveaux, cette observation a déjà été faite, dans des conditions différentes, en pratiquant des infections échelonnées dans le temps : la vitesse de progression de $P$ capsici est plus rapide chez les plantes jeunes ou à maturité qu'à $60 \mathrm{j}$ de culture où la résistance s'exprime au maximum (Pochard et al, 1976; Young et al, 1989). Ces variations de sensibilité de tige peuvent être corrélées à des mesures de conductivité.

Les tissus sains mis en présence de filtrats de culture ou de toxines purifiées donnent des réponses intéressantes. La réaction des tissus se traduit alors par une augmentation de la per- 
méabilité cellulaire que l'on peut mesurer par conductrimétrie. II existe ainsi des différences entre génotypes que l'on a cherché avec plus ou moins de succès à exploiter en sélection pour la résistance vis-à-vis d'un parasite, notamment dans le cas du maïs et de $H$ maydis (Halloin et al, 1973; Pavlovkin et al, 1986; Gerraway et al, 1989), de l'asperge et de Rhizoctonia violacea (Molot et al, 1987), du fraisier et de Rhizoctonia sp (Rosati et al, 1988).

En présence d'un éliciteur, les ions ont tendance à être retenus, peut-être à cause d'une diminution de la perméabilité cellulaire. Cependant, ce résultat n'est pas corroboré par les données bibliographiques. Les rares éliciteurs expérimentés par d'autres auteurs, utilisés sans doute à trop fortes concentrations, engendrent, après mise en contact avec le végétal, un relargage d'ions; ceci est vérifié avec les glycopeptides issus de Fulvia fulva confrontés à une culture cellulaire de feuilles de tomate (Dow et Callow, 1979), les $\beta$-glucanes provenant de $P$ cinnamomi et mis en présence de racines d'eucalyptus (Cahill et al, 1985) ou le chitosane. Ce dernier induit chez Glycine max et Phaseolus vulgaris une libération d'électrolytes qui peut être inhibée par $\mathrm{Ca}^{2+}$ (Young et al, 1982; Young et Kauss, 1983), le même phénomène étant signalé sur endocarpe de pois (Kendra et Hadwiger, 1987). On remarquera cependant, qu'après traitement au chitosane, les cellules sont assez fortement altérées, ce qui expliquerait le relargage d'ions.

Les récents progrès réalisés en biologie animale dans l'étude de la réaction allergique permettent d'apporter quelques éclaircissements sur le mode d'action des phospholipides. Après activation par les complexes immuns allergènes - IgE spécifiques fixés, les phospholipides membranaires des basophiles et des mastocytes modifient la perméabilité cellulaire et déclenchent une entrée de calcium dans la cellule (David, 1988), d'où des mesures conductimétriques traduisant une rétention d'ions. Une migration ionique similaire, indispensable au déclenchement de l'hypersensibilité, est également signalée en pathologie végétale (Atkinson et al, 1990).

Confirmant des travaux antérieurs (Ricci et al, 1988; Molot et al, 1989), nous retrouvons ici une certaine spécificité d'action des éliciteurs purifiés en fonction de la plante hôte : le sphingophospholipide à inositol de $P$ capsici est surtout actif sur piment, la phosphatidylcholine de $G$ graminis sur blé.
Enfin, sans qu'il y ait intervention de toxines ou d'éliciteurs, il devrait être possible, par des mesures conductimétriques effectuées sur du matériel sain, de comparer des génotypes. Des observations intéressantes ont déjà été effectuées sur cultivars de pomme de terre sensibles ou résistants à Erwinia carotovora subsp atroseptica, les tissus des premiers étant caractérisés par un relargage d'ions beaucoup plus important que ceux des seconds (Lewosz et al, 1985).

Ces travaux préliminaires laissent entrevoir l'intérêt des mesures conductimétriques dans la caractérisation de l'état induit par élicitation et dans la recherche de génotypes résistants.

\section{RÉFÉRENCES}

Atkinson MM, Keppler LD, Orlandi EW, Baker CJ, Mischke CF (1990) Involvement of plasma membrane calcium influx in bacterial induction of the $\mathrm{K}^{+} / \mathrm{H}^{+}$and hypersensitive responses in tobacco. Plant Physiol 92, 215-221

Cahil D, Weste, Gretna, Grant B (1985) Leakage from seedling roots inoculated with Phytophthora cinnamoni. Phytopathol Z114, 348-364

David B (1988) Allergie immédiate ou hypersensibilité anaphyllactique. In: Le point sur l'allergie. Influence de l'environnement. CNRS/Piren

Dow JM, Callow JA (1979) Leakage of electrolytes from isolated leaf mesophyll cells of tomato induced by glycopeptides from culture filtrates of Fulvia fulva. Physiol Plant Pathol 15, 27-34

Garraway MO, Akhtar M, Wokoma ECW (1989) Effect of high temperature stress on peroxidase activity and electrolyte leakage in maize in relation to sporulation of Bipolaris maydis race T. Phytopathology 79, 800-805

Halloin JM, Comstock JC, Martinson CA, Tipton CL (1973) Leakage from corn tissues induced by $\mathrm{Hel}$ minthosporium maydis race $\mathrm{T}$ toxin. Phytopathology $63,5,640-642$

Kendra DF, Hadwiger LA (1987) Cell death and membrane leakage not associated with the induction of disease resistance in peas by chitosan or Fusarium f sp phaseoli. Phytopathology 77, 100-106

Lewosz J, Lojkowska E, Holubowska M (1985) Physiological predisposition of potato tubers to soft rot. Ziemniak 49-58

Lhomme O (1988) Analyse des lipides isolés de Phytophthora capsici, inducteurs d'une résistance chez le piment vis-à-vis de ce pathogène. Thèse Fac Sci Lyon, $137 p$

Lhomme O, Bruneteau M, Costello CE, Mas $P$, Molot PM, Dell A, Tiller PR, Michel G (1990) Structural investigations and biological activity of inositol sphin- 
gophospholipids from Phytophthora capsici. Eur $J$ Biochem 191, 203-209

Lucas P, Lemaire JM, Doussinault G, Perraton B, Tivoli B, Carpentier F (1986) Lutte biologique contre Gaeumannomyces graminis (Sacc) Arx et Olivier var tritici (Walker), agent du piétin échaudage, par l'utilisation d'une souche hypoagressive. Résultats. Perspectives. In: Les rotations céréalières intensives. Dix années d'études concertées INRA-ONICITCF. 1973-1983, 113-125

Molot PM (1968) Recherches sur la résistance du maïs à l'Helminthosporiose et aux Fusarioses. Thèse Fac Sci Bordeaux. $239 p$

Molot PM, Clerjeau M, Nourrisseau JG, Ricci P (1976) La résistance du piment à Phytophthora capsici. III. Étude, sur extraits de tiges sensibles et résistantes, du pouvoir antifongique induit par la contamination. Ann Phytopathol 8 (4), 399-409

Molot PM, Mas $P$, Conus $M$, Ferrière H (1980a) La résistance du piment à Phytophthora capsici. VII. Protection des organes foliaires après mise en survie sur une filtrat de culture du parasite. Caractérisation des conditions d'expression du phénomène. Ann Phytopathol 12 (2), 97-107

Molot $\mathrm{P}$, Staron T, Mas $\mathrm{P}$ (1980b) La résistance du piment à Phytophtora capsici. VIll. Induction de résistance et de capsidiol chez Capsicum annuum avec des fractions obtenues à partir des filtrats de cultures et du mycélien de Phytophthora capsici. Ann Phytopathol 12 (4), 379-387

Molot PM, Ferrière $H$, Conus M (1987) Mesure de la sensibilité de l'asperge à l'action des toxines de $R$ hizoctonia violacea. Rev Cytol Biol Végét 10, 147-154

Molot PM, Mas P, Burgerjon-Glandard A, Denizot Y, Staron T, Lhomme O, Bruneteau M, Lemaire JM (1989) Essais préliminaires d'application d'éliciteurs fongiques sur diverses plantes. $1^{\text {er }}$ Congrès SFP, $\mathrm{N}^{\circ}$ spécial. Sci Agron Rennes, 53-54
Newton HC, Maxwell DP, Sequeira L (1973) A conductivity assay for measuring virulence of Sclerotinia sclerotiorum. Phytopathology 63, 424-428

Pavlovkin J, Mistrik I, Zajcenko AM (1986) Effect of mycotoxins on the ultrastructure and permeability of primary maize root cell membranes. Biologia 41, $11,1085-1089$

Pochard E, Clerjeau M, Pitrat M (1976) La résistance du piment (Capsicum annuum) au Phytophthora capsici. I. Mise en évidence d'une induction progressive de la résistance. Ann Amélior Plant 28 (1), 35-50

Ricci $\mathrm{P}$, Bonnet $\mathrm{P}$, Ponchet $\mathrm{M}$, Molot PM, Bruneteau $M$ (1988) Recherche et intérêt d'éliciteurs fongiques dans les interactions de 3 espèces végétales avec des champignons phytopathogènes du genre Phytophthora. Cryptog Mycol 9, 191-200

Rosati P, Gaggioli D, Mezzetti B (1988) Screening for strawberry resistant to Rhizoctonia fragariae and $R$ solani using the cultural filtrate of the pathogen. Int Strawberry Symp, Cesena, May 1988, book of abstracts, 99

Young DH, Kauss H (1983) Release of calcium from suspension-cultured Glycine max cells by chitosan, other polycations and polyamines in relation to effects on membrane permeability. Plant Physiol 73, 698-703

Young DH, Kohle H, Kauss H (1982) Effect of chitosan on membrane permeability of suspension cultured Glycine max and Phaseolus vulgaris cells. Plant Physiol 70, 1449-1454

Young Jin Kim, Byung Kook Hwang, Kuen Woo Park (1989) Expression of age-related resistance in pepper plants infected with Phytophthora capsici. Plant Dis 73, 9, 745-747

Zilberstein M, Pinkas $Y$ (1987) Detached root inoculation. A new method to evaluate resistance to Phytophthora root rot in avocado trees. Phytopathology $77,841-844$ 